\title{
FACTORES ASOCIADOS A LA REDUCCIÓN DE LA HEMOGLOBINA EN PUÉRPERAS ATENDIDAS EN EL INSTITUTO NACIONAL MATERNO PERINATAL - 2012
}

\author{
Clara Díaz Tinoco ${ }^{1}$
}

\begin{abstract}
RESUMEN
Objetivo. Analizar los factores que se asocian a la reducción de los niveles de hemoglobina en puérperas atendidas en el Instituto Nacional Materno Perinatal durante el año 2012. Materiales y Métodos. Estudio de casos y controles donde se asignó a 274 pacientes puérperas divididas en 137 con diferencia de hemoglobina $\geq 1,3 \mathrm{~g} / \mathrm{dl}$ (casos) y 137 con diferencia de hemoglobina $<1,3 \mathrm{~g} / \mathrm{dl}$ (controles). Se aplicó estadísticas descriptivas e inferenciales, medidas de tendencia central y de dispersión en las variables cuantitativas, distribución de frecuencias para las variables cualitativas, para las inferencias estadísticas se aplicó Chi cuadrado, OR IC al 95\% y regresión logística binaria para el análisis multivariado. Resultados. Podemos determinar que se presentaron diferencias significativas para el estado civil, siendo de estado civil soltera en $19,7 \%$ de los casos y de $8 \%$ para los controles. El 2,2\% de los casos presentó multiparidad, comparado con el 10,2\% de los controles. El $59,1 \%$ de los casos presentó episiotomía, comparado con el $35,8 \%$ de los controles, existiendo 2,6 veces mayor probabilidad de tener una reducción marcada de la hemoglobina cuando se tiene episiotomía (OR: 2,6 IC95\% 1,6:4,2). No se encontró asociación con factores nutricionales, patológicos, factores posparto. El análisis multivariado encontró riesgo para administración de sulfato de magnesio, retención de restos placentarios, episiotomía, soltera, menarquia ( 8 a 13 años) y multiparidad (>4 partos). Conclusión. La administración de sulfato de magnesio, retención de restos placentarios, episiotomía, soltera, menarquia (8 a 13 años) y multiparidad (>4 partos) se asociaron a la reducción de la hemoglobina posparto.
\end{abstract}

Palabras Clave: Hemoglobina; Posparto; Gestante (Fuente: DeCS BIREME).

\section{FACTORS ASSOCIATED TO THE HEMOGLOBIN REDUCTION IN POSTPARTUM WOMEN TREATED AT THE MATERNAL PERINATAL NATIONAL INSTITUTE-2012}

\begin{abstract}
Objective. To analyze the factors that are associated with reduced hemoglobin levels in postpartum women treated at the National Maternal and Perinatal Institute during 2012. Materials and methods. Case-control study where 274 patients divided in 137 postpartum women with hemoglobin $\geq$ difference $1.3 \mathrm{~g} / \mathrm{dl}$ (cases) and 137 with a difference of hemoglobin $<1.3 \mathrm{~g} / \mathrm{dl}$ (controls). Descriptive and inferential statistics, measures of central tendency and dispersion for quantitative variables, frequency distribution for qualitative variables for statistical inferences applied Chi-square, $\mathrm{OR} 95 \% \mathrm{Cl}$ and binary logistic regression for multivariate analysis was applied. Results. We can determine significant differences for marital status were presented, being unmarried marital status in $19.7 \%$ of cases and $8 \%$ for controls. $2.2 \%$ of the cases presented multiparity, compared with $10.2 \%$ of controls. $59.1 \%$ of the patients had episiotomy, compared with $35.8 \%$ of controls , having 2.6 times more likely to have a marked reduction in hemoglobin when you have episiotomy (OR: 2,6 CI95\% 1,6:4,2). Not association with nutritional factors, pathological factors found postpartum. Multivariate analysis found risk management magnesium sulfate, retained placental remnants, episiotomy, single, menarche (8-13 years) and multiparity ( $>4$ births). Conclussion. The administration of magnesium sulfate, retained placental remnants, episiotomy, single, menarche (8-13 years) and multiparity (> 4 births) were associated with the reduction of postpartum hemoglobin.
\end{abstract}

Key Words: Hemoglobin; Postpartum; Pregnant (Source:MeSH NLM).

\section{INTRODUCCIÓN}

La anemia es la más frecuente de las enfermedades que puede coincidir con el embarazo o ser producidas por este. Según estudios de prevalencia realizados en diferentes regiones del mundo, se presenta entre el 30 y el $70 \%$ de las gestantes. Estudios clínicos revelaron que la anemia se asocia con complicaciones del embarazo y del parto en la madre, en el feto y el recién nacido, como mayor morbilidad y mortalidad fetal y perinatal, así como bajas reservas de hierro en el recién nacido, lo que provoca desarrollo psicomotor retardado y alteraciones neuroconductuales. La mayoría de las mujeres que inician el embarazo con $\mathrm{Hb}$ entre 11 a $11,9 \mathrm{~g} / \mathrm{dl}$ desarrollan anemia en el segundo o tercer trimestre de gestación ${ }^{1,2}$.

Debido a su importancia, la hemorragia durante el parto es considerada dentro de los indicadores de calidad 
asistencial para la Sociedad Española de Ginecología y Obstetricia (SEGO). Entre los factores que predisponen a un mayor sangrado podemos destacar: la edad materna avanzada, multiparidad, antecedentes de hemorragia puerperal en gestaciones previas, sobre distensión uterina, alteración de la contractilidad uterina, parto prolongado o precipitado, coagulopatía materna, complicaciones que se asocian con hemorragias anteparto (placenta previa, desprendimiento prematuro de la placenta normoinserta), partos instrumentales, inducción de parto, los desgarros de diverso grado y la realización de episiotomía.

En nuestra institución, que es una institución del Ministerio de Salud, atiende a más de 16 mil partos al año, muchos de ellos con anemia, y en muchos de los casos, la anemia se agudiza posterior al parto, por lo que el propósito de la investigación es identificar los factores que mayor probabilidad tienen de relacionarse con la anemia aguda posparto.

\section{MATERIAL Y MÉTODOS}

El presente estudio es de tipo prospectivo, transversal, analítico.

El diseño es de casos y controles.

Universo de pacientes que acuden a la institución:Total de puérperas atendidas en el Instituto Nacional Materno Perinatal.

Población a estudiar:Puérperas atendidas en el Instituto Nacional Materno Perinatal para su parto durante el año 2012.

\section{Muestra de estudio o tamaño muestral}

Unidad de análisis: Puérpera atendidas en el Instituto Nacional Materno Perinatal durante el año 2012.

\section{Tamaño de la muestra:}

El tamaño de la muestra fue de 274 pacientes puérperas divididas en 137 con diferencia de hemoglobina $\geq 1,3 \mathrm{~g} /$ dl (casos) y 137 con diferencia de hemoglobina $<1,3 \mathrm{~g} / \mathrm{dl}$ (controles) pareadas por fecha de atención y procedencia de la madre de la puérpera.

Tipo de muestreo: Se aplicó la técnica de muestreo no probabilístico apareado.

\section{Técnica y método de trabajo}

Se aplicaron las siguientes técnicas:

- Para la revisión de las historias clínicas y los resultados de laboratorio se aplicó la observación documental.

- Para la determinación de algunos factores de riesgo y datos obstétricos, reproductivos y generales se aplicó la entrevista estructurada.

\section{Criterios de inclusión}

- Para los casos

- Aceptó participar en el estudio

Diferencia de hemoglobina $\geq 1,3 \mathrm{~g} / \mathrm{dl}$

Dosaje de hemoglobina en tercer trimestre

Dosaje de hemoglobina

- Para los controles

- Aceptó participar en el estudio

Diferencia de hemoglobina $<1,3 \mathrm{~g} / \mathrm{dl}$

Dosaje de hemoglobina en tercer trimestre

- Dosaje de hemoglobina

\section{Criterios de exclusión}

- Para ambos grupos

- Traslado de paciente

- Fallecimiento de recién nacido

- Diagnóstico de hemorragia posparto

Análisis de los datos. Se aplicó el programa estadístico SPSS versión 20.0 para el análisis de datos, se aplicó estadísticos descriptivos para las estimaciones. En las variables cualitativas se aplicó la distribución de frecuencias y en las variables cuantitativas medidas de tendencia central como la media y mediana, así como medidas de dispersión como la desviación estándar, el rango y la varianza. Por ser un muestreo no probabilístico apareado, se aplicó coeficientes de asimetría para la determinación de estadísticos. Para las inferencias estadísticas se aplicó en las variables cualitativas en estadístico Chi cuadrado, en las variables cuantitativas se aplicó t-student para la diferencia de medias ambos con un nivel de significación estadístico $p<0,05$. Para la determinación de las asociaciones se aplicó la razón de productos cruzados (OR) con sus intervalos de confianza al $95 \%$, así mismo para el análisis multivariado se aplicó la regresión logística binaria, con el método paso a paso, entre las variables factores y los casos y controles, considerándose significativo a un nivel de significación estadístico $p<0,05$.

\section{RESULTADOS}

\section{Factores nutricionales}

No se encontró diferencias significativas entre casos y controles con la malnutrición crónica, ingesta dietética inadecuada, anemia previa, déficit de micronutrientes y obesidad (Tabla 1).

\section{Factores patológicos}

No se encontró diferencias significativas entre casos y controles con el diagnóstico de hepatitis y fiebre (Tabla 2). No se encontraron casos de las otras patologías investigadas como malaria, paludismo, $\mathrm{VIH}$, entre otros en el periodo de estudio. 
Tabla 1. Factores nutricionales asociados a la reducción de hemoglobina - instituto nacional materno perinatal, 2012

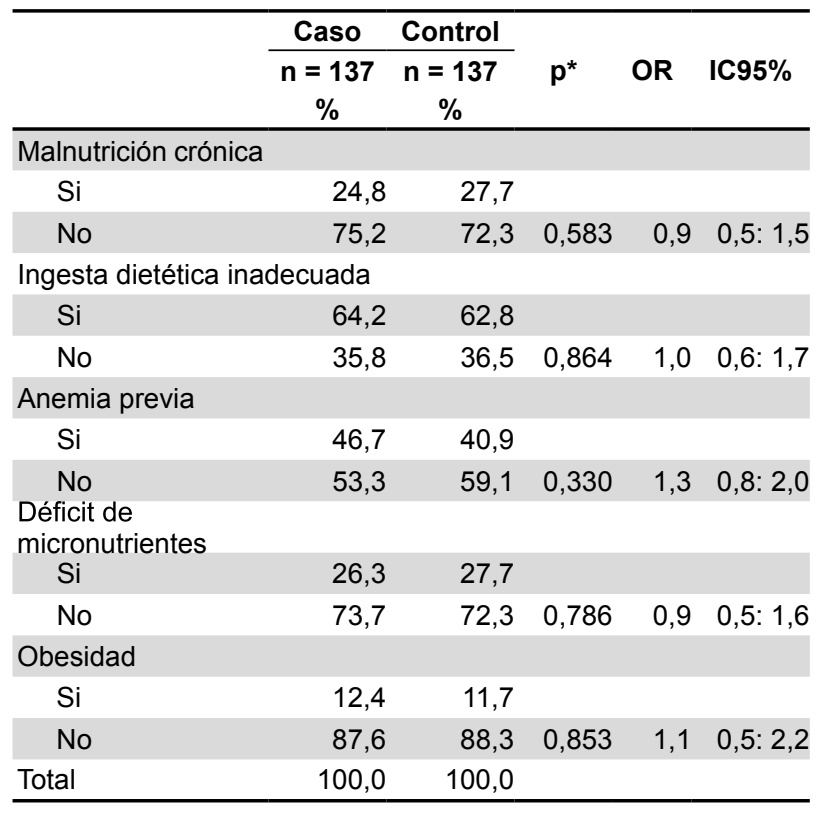

* Significación estadística con prueba Chi-cuadrado

Tabla 2. Factores patologicos asociados a la reducción de hemoglobina - instituto nacional materno perinatal, 2012

\begin{tabular}{|c|c|c|c|c|}
\hline & Caso & Control & & \\
\hline & $\begin{array}{c}\mathrm{n}=137 \\
\%\end{array}$ & $\begin{array}{c}n=137 \\
\%\end{array}$ & $\mathbf{p}^{*}$ & OR IC95\% \\
\hline \multicolumn{5}{|c|}{ Diagnóstico de hepatitis } \\
\hline $\mathrm{Si}$ & 0,7 & 0,7 & & \\
\hline No & 99,3 & 99,3 & 1,000 & $1,00,1: 16,2$ \\
\hline \multicolumn{5}{|l|}{ Fiebre } \\
\hline $\mathrm{Si}$ & 7,3 & 8,0 & & \\
\hline No & 92,7 & 92,0 & 0,820 & $0,8 \quad 0,4: 2,2$ \\
\hline Total & 100,0 & 100,0 & & \\
\hline
\end{tabular}

*Significación estadística con prueba Chi-cuadrado

\section{Factores anteparto}

No se encontraron diferencias significativas entre casos y controles para el periodo intergenésico menor a 2 años, Preeclampsia, diagnóstico de miomatosis, parto pretérmino, cesáreas previas, cirugías durante el puerperio, diagnóstico de macrosomía fetal, administración de sulfato de magnesio y antecedente de cesárea anterior. Se presentaron diferencias significativas para la multiparidad (>4 partos) y primiparidad. El $2,2 \%$ de los casos presentó multiparidad, comparado con el 10,2\% de los controles, considerándose que cuando no se es multípara, la probabilidad de reducción en la probabilidad de reducción de la hemoglobina en el posparto se reduciría en un 80\% (OR: 0,2 IC95\% 0,1:0,7). Así mismo el $52,6 \%$ de los casos fueron primíparas, comparado con el $36,5 \%$ de los controles, existiendo 1,9 veces mayor probabilidad de tener una reducción de la hemoglobina marcada cuando se es primípara que cuando no lo es (OR: 1,9 IC95\% 1,2:3,1); (Tabla 3).

Tabla 3. Factores anteparto asociados a la reducción de hemoglobina - instituto nacional materno perinatal, 2012

\begin{tabular}{|c|c|c|c|c|c|}
\hline & Caso & Control & & סת & IC050\% \\
\hline & $n=137$ & $n=137$ & & & \\
\hline Menarquia (años) & & & & & \\
\hline 8 a 13 & 48,2 & 62,8 & & & \\
\hline 14 a 18 & 51,8 & 37,2 & 0,015 & 0,6 & $0,3: 0,9$ \\
\hline Periodo intergenésico & ños & & & & \\
\hline $\mathrm{Si}$ & 10,9 & 10,9 & & & \\
\hline No & 89,1 & 89,1 & 1,000 & 1,0 & $0,5: 2,1$ \\
\hline Preeclampsia & & & & & \\
\hline $\mathrm{Si}$ & 4,4 & 5,1 & & & \\
\hline No & 95,6 & 94,9 & 0,776 & 0,9 & $0,3: 2,6$ \\
\hline Diagnóstico de mioma & & & & & \\
\hline $\mathrm{Si}$ & 0,7 & 0,7 & & & \\
\hline No & 99,3 & 99,3 & 1,000 & 1,0 & $0,1: 16,2$ \\
\hline Diagnóstico de parto $p$ & $\operatorname{nino}$ & & & & \\
\hline $\mathrm{Si}$ & 3,6 & 2,9 & & & \\
\hline No & 96,4 & 97,1 & 0,735 & 1,3 & $0,3: 4,8$ \\
\hline Cesárea de urgencia & 0,0 & 0,0 & & & \\
\hline $\mathrm{Si}$ & 7,3 & 10,2 & & & \\
\hline No & 92,7 & 89,8 & 0,393 & 0,7 & $0,3: 1,6$ \\
\hline Multiparidad ( $>4$ parto & & & & & \\
\hline $\mathrm{Si}$ & 2,2 & 10,2 & & & \\
\hline No & 97,8 & 89,8 & 0,010 & 0,2 & $0,1: 0,7$ \\
\hline Primiparidad & & & & & \\
\hline $\mathrm{Si}$ & 52,6 & 36,5 & & & \\
\hline No & 47,4 & 63,5 & 0,007 & 1,9 & $1,2: 3,1$ \\
\hline Cirugía durante el pue & & & & & \\
\hline $\mathrm{Si}$ & 2,9 & 1,5 & & & \\
\hline No & 97,1 & 98,5 & 0,684 & 2,0 & $0,4: 11,3$ \\
\hline Diagnóstico de macros & fetal & & & & \\
\hline $\mathrm{Si}$ & 5,1 & 8,8 & & & \\
\hline No & 94,9 & 91,2 & 0,234 & 0,6 & $0,2: 1,5$ \\
\hline Administración de sulf & magnes & & & & \\
\hline $\mathrm{Si}$ & 4,4 & 0,7 & & & \\
\hline No & 95,6 & 99,3 & 0,120 & 6,2 & $0,7: 52,4$ \\
\hline Antecedentes de cesá & nterior & & & & \\
\hline $\mathrm{Si}$ & 9,5 & 8,8 & & & \\
\hline No & 90,5 & 91,2 & 0,834 & 1,1 & $0,5: 2,5$ \\
\hline Total & 100,0 & 100,0 & & & \\
\hline
\end{tabular}

* Significativo con prueba de Chi cuadrado o Exacto de Fischer

\section{Factores del parto}

El 59,1\% de los casos presentó episiotomía, comparado con el $35,8 \%$ de los controles, existiendo 2,6 veces mayor probabilidad de tener una reducción marcada de la hemoglobina cuando se tiene episiotomía (OR: 2,6 IC95\% 1,6:4,2). EI 34,9\% de los casos presentó inducción o estimulación con oxitocina, comparado con el $21,9 \%$ de los controles, existiendo 1,9 veces mayor probabilidad de tener una reducción marcada de la hemoglobina cuando se tiene inducción o estimulación con oxitocina (OR: 1,9 IC95\% 1,1:3,2) (Tabla 4). 
Tabla 4. Factores de parto asociados a la reducción de hemoglobina - Instituto Nacional Materno Perinatal, 2012

\begin{tabular}{|c|c|c|c|c|c|}
\hline & Caso & Control & & & \\
\hline & $n=137$ & $n=137$ & p & UR & $1495 \%$ \\
\hline Lacera & & & & & \\
\hline $\mathrm{Si}$ & 5,8 & 7,3 & & & \\
\hline No & 94,2 & 92,7 & 0,626 & 0,8 & $0,3: 2,1$ \\
\hline Lacera & & & & & \\
\hline $\mathrm{Si}$ & 13,9 & 14,6 & & & \\
\hline No & 86,1 & 85,4 & 0,863 & 0,9 & $0,5: 1,9$ \\
\hline Episiot & & & & & \\
\hline $\mathrm{Si}$ & 59,1 & 35,8 & & & \\
\hline No & 40,9 & 64,2 & $<0,001$ & 2,6 & $1,6: 4,2$ \\
\hline Analge & & & & & \\
\hline $\mathrm{Si}$ & 34,3 & 24,8 & & & \\
\hline No & 65,7 & 75,2 & 0,085 & 1,6 & $0,9: 2,7$ \\
\hline Inducci & n con oxi & kitocina & & & \\
\hline $\mathrm{Si}$ & 34,3 & 21,9 & & & \\
\hline No & 65,7 & 78,1 & 0,022 & 1,9 & $1,1: 3,2$ \\
\hline Trabajc & gado $(>1$ & 12 horas) & & & \\
\hline $\mathrm{Si}$ & 0,7 & 0,7 & & & \\
\hline No & 99,3 & 99,3 & 1,000 & 1,0 & $0,1: 16,2$ \\
\hline Medica & s uterino & & & & \\
\hline $\mathrm{Si}$ & 7,3 & 14,6 & & & \\
\hline No & 92,7 & 85,4 & 0,053 & 0,5 & $0,2: 1,0$ \\
\hline Total & 100,0 & 100,0 & & & \\
\hline
\end{tabular}

* Valor $p$ con prueba de Chi cuadrado

\section{Factores posparto}

No se encontraron diferencias significativas con los factores posparto como retención de restos placentarios, desgarro cervical o desgarro vaginal entre casos y controles (Tabla 5).
Tabla 5. Factores posparto asociados a la reducción de hemoglobina (casos) - Instituto Nacional Materno Perinatal, 2012

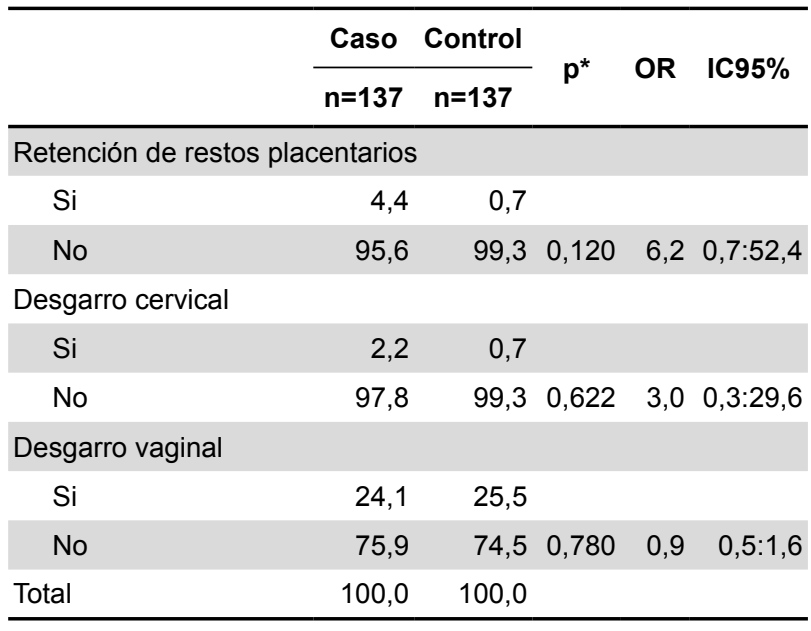

* Significativo con prueba Chi-cuadrado o Exacto de Fisher

\section{Modelo de regresión logística}

Se plantea un modelo de factores asociados a la reducción de la hemoglobina posparto, para el ajuste global del modelo, se aplicó la prueba de Hosmer y Lemeshow, el cual nos indicó el modelo propuesto fue adecuado $(p=0,836)$ así mismo el $R^{2}$ de Cox y Snell nos indicó que el $15,5 \%$ de la variación de la reducción de la hemoglobina posparto, es explicada por la los factores administración de sulfato de magnesio, retención de restos placentarios, episiotomía, ser soltera, menarquia de 8 a 13 años y multiparidad ( $>4$ partos). Los factores administración de sulfato de magnesio, retención de restos placentarios, episiotomía, ser soltera y menarquia entre 8 a 13 años se consideraron de riesgo para reducción de hemoglobina posparto, mientras que el factor no ser multípara se comportó protector (Tabla 6). El presente modelo explica el $71,6 \%$ de la varianza del evento (IC 95\% 0,656:0,776); (Figura 1).

Tabla 6. Factores asociados a la reducción de hemoglobina, instituto nacional materno perinatal, 2012

\begin{tabular}{lrrrrrrrrr}
\hline & B & \multirow{2}{*}{ Error Est. } & Wald & gl & Sig. & Exp(B) & \multicolumn{2}{c}{ IC 95\% Exp(B) } \\
\cline { 5 - 10 } & & & & & & & & L. Inf. & L. Sup. \\
\hline Administración Sulfato de Magnesio & 3,179 & 1,290 & 6,137 & 1 & 0,013 & 24,5 & 2,0 & 306,8 \\
Retención de restos placentarios & 2,622 & 1,29, & 4,115 & 1 & 0,043 & 13,8 & 1,1 & 173,5 \\
Episiotomía & 0,990 & 0,266 & 13,803 & 1 & $<0,001$ & 2,7 & 1,6 & 4,5 \\
Soltera & 0,812 & 0,398 & 4,157 & 1 & 0,041 & 2,3 & 1,03 & 4,91 \\
Menarquia (8-13 años) & 0,548 & 0,266 & 4,258 & 1 & 0,039 & 1,7 & 1,03 & 2,91 \\
Multiparidad (>4 partos) & $-1,902$ & 0,842 & 5,106 & 1 & 0,024 & 0,2 & 0,03 & 0,78 \\
Constante & $-0,950$ & 0,255 & 13,849 & 1 & $<0,001$ & 0,4 & & \\
\hline
\end{tabular}

Prueba de Hosmer y Lemeshow $=2,778 \mathrm{gl}=6 \mathrm{p}=0,836$

$R^{2}$ Cox y Snell $=0,155$ 


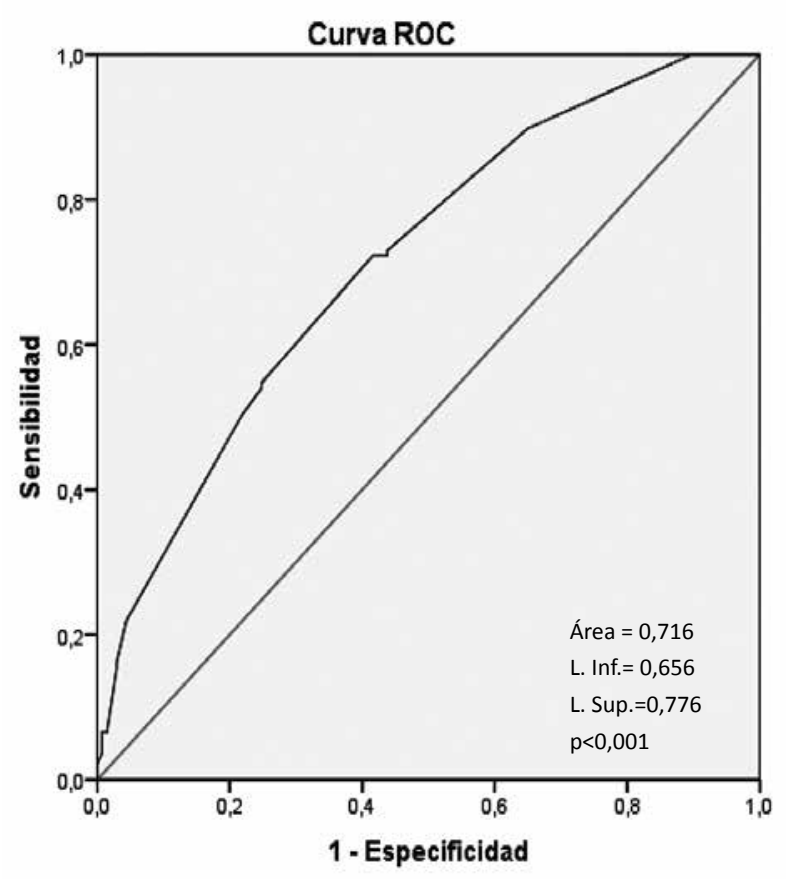

Figura 1. Curva ROC de las probabilidades del modelo de regresión logística binaria de los factores asociados a la reducción de la hemoglogina.

\section{DISCUSIÓN}

La presente investigación nos ha permitido encontrar que existen factores que se asocian a la reducción de la hemoglobina posparto, entre ellas tenemos a factores anteparto, parto y del posparto. Entre los factores anteparto tenemos a ser soltera, menarquia temprana (8 a 13 años) y multiparidad (>4 partos); entre los factores del parto la administración de sulfato de magnesio y la episiotomía y en los factores posparto la retención de restos placentarios.

Un evento que se ha podido determinar es que los factores encontrados para esta reducción de la hemoglobina, sean similares a los factores que se asocian a la hemorragia por parto, esto quiere indicar que existiría una conexión marcada entre los factores de la reducción de la hemoglobina con los factores de la hemorragia posparto, y con ello a una de las primeras causas de muerte materna en el mundo. En un estudio realizado en Argentina (2005) se encontró que entre los factores de riesgo durante el embarazo para hemorragia posparto fueron: primigestas (32\%), Rotura Prematura de las Membranas (16\%), edad extrema (<18 o >35 años) con 16\%, gran multípara (15\%) y anemia $(14,7 \%)^{3}$.

Se encontró que el no ser multípara se asoció a no tener reducción de hemoglobina posparto, es decir, que cuando la parturienta no es multípara ( $>$ partos) es menos probable que termine con una reducción marcada o pérdida de hemoglobina en el posparto. Lo que se ha podido indicar aquí, que la no multiparidad, que es un evento de desgaste desde el punto de vista obstétrico, porque expone a la madre a lesiones no solo en el parto, sino en el posparto y a una pérdida de la elasticidad de las fibras musculares, provocando así mayor probabilidad de sangrado y por ende de pérdida de hemoglobina. Factores de riesgo que se asocian a mayor incidencia de hemorragia posparto como la sobredistensión del útero debida a polihidramnios, embarazo múltiple o macrosomía fetal, la gran multiparidad, el trabajo de parto prolongado, especialmente el asociado a inducción oxitócica, historia previa de hemorragia posparto y síndrome hipertensivo del embarazo, así como la administración de anestesia general, implantación anormal de la placenta y parto operatorio 4 .

Entre los factores del parto, se ha determinado que existe 24,5 veces mayor probabilidad de presentar reducción de hemoglobina en el puerperio cuando se ha administrado sulfato de magnesio a la gestante. El sulfato de magnesio inhibe la actividad miometrial, compitiendo con el calcio para entrar a través de los canales de calcio al miocito, lo que reduce la frecuencia de la despolarización del músculo liso a través de la modulación de la captación, fijación y distribución del calcio en las células del músculo liso $^{5}$. Este fármaco, generalmente se administra a las parturientas cuando se presenta estados hipertensivos en el embarazo, o para prevención de convulsiones por lo que se puede determinar que el mismo haya sido empleado en situaciones de riesgo tanto para la madre como para el feto, por ello el manejo con este fármaco siempre será sopesando la razón riesgo/beneficio, es decir, se prima los posibles riesgos para la madre, como es la reducción de hemoglobina o pérdida sanguínea contra el beneficio que sería la prevención de convulsiones para la madre y para el feto.

En la presente investigación se ha podido determinar que existe 2,7 veces mayor probabilidad de presentar reducción de la hemoglobina en el posparto cuando se ha practicado una episiotomía. El objetivo de la episiotomía es sustituir el desgarro desigual por uno fácil de reparar, previniendo la relajación pélvica, cistocele, rectocele y la incontinencia urinaria. La pérdida sanguínea por episiotomía puede alcanzar una media de unos $200 \mathrm{ml}$ a $250 \mathrm{ml}^{6}$. También se puede indicar que este es un factor que predispone a hemorragia en el posparto. Rockner et al (citado por Mejía y colaboradores) definen la episiotomía como un corte quirúrgico del cuerpo perineal que se práctica para proteger el esfínter y el recto de laceraciones o desgarros severos durante el parto, como también para disminuir la duración de la segunda etapa del mismo y prevenir la aparición de futuros prolapsos genitales ${ }^{7}$. Si la episiotomía se realiza demasiado pronto, la hemorragia por la herida puede ser considerable durante el tiempo que transcurre entre la incisión y el nacimiento del niño ${ }^{8}$. Estudios han identificado que la práctica de la episiotomía incrementa el riesgo de sangrado excesivo en 4,8 veces (OR: 4,8 IC: $95 \%$ 1,73-13,44) ${ }^{9}$. Iglesias y colaboradores encontró que el descenso de hemoglobina cuando se practica la episiotomía es de 1,57 $\pm 1,11 \mathrm{~g} / \mathrm{dl}$ y de $1,09 \pm 0,9 \mathrm{~g} / \mathrm{dl}^{6}$. 
Se ha podido determinar que existe 1,8 veces mayor probabilidad de reducción de hemoglobina mayor a 1,3 g/ $\mathrm{dl}$ en aquellas que han presentado retención de restos placentarios, este evento ocurre cuando uno o más lóbulos placentarios quedan retenidos, lo cual impide que el útero se contraiga eficazmente ${ }^{10}$. La contracción y la retracción uterina empiezan luego de la expulsión de la placenta y su correcta expulsión permite una contracción y oclusión de los vasos sanguíneos respectivos. La retención de una porción de la placenta es más común si la placenta se desarrolla como succenturiada o con un lóbulo accesorio, normalmente, después de la expulsión de la placenta esta debe ser examinada; más aún, si luego de su expulsión existe sangrado, la placenta debería obligatoriamente ser inspeccionada, para evidenciar la integridad de los vasos sanguíneos y la integridad de las membranas, estos hallazgos clínicos sugieren una retención parcial o un lóbulo accesorio. Esto es más frecuente en gestaciones pretérmino extremos (menor de 24 semanas) y por ello está normada la revisión de la cavidad uterina en estos casos ${ }^{11}$. La retención de tejido placentario y membranas de la placenta es la causa de 5 a $10 \%$ de las hemorragias posparto ${ }^{12}$.

\section{REFERENCIAS BIBLIOGRÁFICAS}

1. Villares I, Fernández J, Avilés M, Mediaceja O, Guerra T. Anemia y deficiencia de hierro en embarazadas de un área urbana del municipio de Cienfuegos. Rev Cuba Obstet Ginecol 2006; 32(1).

2. Iglesias-Benavides J, Tamez-Garza L, Reyes-Fernández I. Anemia y embarazo, su relación con complicaciones maternas y perinatales. Medicina Universitaria 2009; 11(43):95-98.
3. Rivero M, Avanza M, Alegre M, Feu M, et al. Hemorragia postparto: incidencia, factores de riesgo y tratamiento. (En línea) dirección URL disponible en http://www.unne.edu.ar/ unnevieja/Web/cyt/com2005/3-Medicina/M-106_Falta\%20 Corregir.pdf acceso 27-04-2014.

4. Pérez A, Donoso E. Pérez Sánchez Obstetricia. 2da. Ed. Santiago. Mediterraneo. 1997.

5. Ortiz M, Labrador A, Ayala R. Uteroinhibidores actuales para el manejo de parto pretérmino. An Med (Mex) 2010; 55(2):85-91.

6. Iglesias S, Montenegro M, González M, Conde M. Factores perinatales que influyen en el descenso de hemoglobina materna tras el parto y en el $\mathrm{pH}$ neonatal. Matronas Prof 2011; 12(4):97-103.

7. Mejía M, Quintero V, Tovar M. ¿La episiotomía debe ser un procedimiento de rutina? Colomba Med 2004; 35(2):75-81.

8. Cunningham $G$, MacDonald $P$, Gant $N$, Leveno $K$, et al William's Obstetricia. $20^{\circ}$ Ed. Panamericana. Buenos Aires. 1998.

9. Romero-Arias A, Luján-Prior $M$, Pernia-Fernández J, Hernández-Martínez A. Incidencia y factores relacionados con la pérdida hemática intraparto excesiva. Rev Enferm Clin 2011; 24(5):256-63.

10. Ortiz A, Miño L, Ojeda P, Medina S, Iratí G. Hemorragia puerperal. Rev Posgrado Vla Cátedra Med 2011; 206:1620.

11. Calle A, Barrera B, Guerrero A. Diagnóstico y manejo de la hemorragia posparto. Rev Per Ginecol Obstet. 2008; 54:233243.

12. Chaparro C, LutterCh.Laanemia entreadolescentesymujeres adultas jóvenes en América Latina y El Caribe. Organización Panamericana de la Salud. Un motivo de preocupación. (En línea) dirección URL disponible en http://new.paho.org/hq/ dmdocuments/2009/AdolescentAnemiaSpan $\% 20 \% 282 \% 29$. pdf Acceso 18-08-2012. 